\title{
Smartphone citizen science: can a conservation hypothesis be tested using non specialist technology?
}

\author{
T. Wess ${ }^{*}$ (D)
}

\begin{abstract}
The work presented here, describes a citizen science scoping study using accessible stand-alone smartphone technology. Paper discolouration in a single journal type, the Wagga Wagga Daily Advertiser published from the dates 1876-2004 was chosen as the focus of study, with a specific hypothesis to be tested; that the wartime journals were more discoloured by yellowing due to them being accessed more frequently. A series of measurements were carried out with a smartphone device using the camera to act as a colorimeter and converting the CIE $L$ xy vector length into a yellowing parameter $Y$. A number of preliminary attempts were made to recreate conditions that corresponded to the measurements of $Y$ using a conventional spectrophotometer, this was by standardising distance from object and through a number of lighting conditions. It was found that the most consistently comparable results (with an offset) could be obtained using an iPhone $6 \mathrm{~S}$ with the light source from an additional iPhone with sample to aperture distance of $4 \mathrm{~cm}$. Both studies showed that the large increase in Y occurred in journals printed around 1912 with a plateau of elevated $Y$ value detected in journals until the 1950s after which the $Y$ parameter decreased until 2004. This indicates the frequency access hypothesis to be false and this negative proof was able to be evidenced by the data collected by the iPhone. It would be suggested that great care needs to be taken if such an approach of stand-alone measurement were to be taken into citizen science; training and testing would need to be undertaken, because of the challenge of reproducibility and the risk of sampling irreplaceable objects.
\end{abstract}

\section{Background}

Citizen science is a sub group of crowdsourcing, the outsourcing of a function by a company or institution to an undefined and generally large network of people. Although far from being a new phenomenon, it has come to the fore recently because the tools to hand for analysis, combined with digital interconnectedness means that more people can contribute to scholarly work [1]. Modern technology has allowed major advances to be made in cultural heritage research that allows greater accessibility through improvements in the non-invasive sampling of artefacts. The affordability of technology also has the potential to put technology for research in the hands of everyone as potential citizen scientists or researchers.

\footnotetext{
*Correspondence: twess@csu.edu.au

Faculty of Science, Charles Sturt University, Booroma Street, Wagga Wagga, NSW, Australia
}

The fact that over $80 \%$ of the population in Australia now owns some kind of smartphone means that community engagement projects that utilize smartphone and tablet technology are within reach to many disciplines with citizen based contributions to mainstream scientific investigations becoming increasingly important. Technology is helping to make projects more accessible to the public, providing greater effectiveness of communications between science practitioners and citizen scientists, and providing accessible data collection and management tools which improve the flow and quality of data.

For example in the Atlas of Living Australia [2] "Citizen science is a very important source of data about biodiversity. Data and insights gained through the efforts of citizen scientists can be as valuable as those obtained by scientists working in academia, natural history collections, government agencies and business. Harnessing the enthusiasm, interest and efforts of the thousands of 
people participating in citizen science will continue to enhance the range and depth of data available for analysis and research."

Many citizen science projects relate to biodiversity observation, with fewer projects relating to conservation/curation of historical objects or measuring change in historical objects. The projects related to art conservation and digital conservation tracking such as ARTigo [3], speak to the gamification of data capture and the attachment of meta data to digitally captured but not necessarily curated artwork [4]. A smaller subset within conservation of cultural heritage speak to crowdsourced measurement/inspection of the state of collections $[5,6]$.

Beyond the use of smartphones to capture images, the digital camera has functionality to act as a colorimeter, where the field of view is converted to several quantified colour outputs that can be utilised by anyone interested in citizen science. Duplication and reliability are often a common causes of concern in the engagement of the non expert, however the challenged posed here was to respond to a hypothesis put by the manager of the Wagga Wagga archive, and to see if this could be tested, using the stand-alone technological capability of a smartphone.

The conception of the work described here arose from a conversation of the researcher with the archivist at the Wagga Wagga Riverina Regional Archive. On discussion of the yellowing of the archive copies of the local paper, the Wagga Wagga Daily Advertiser, it was posited that the archived editions of the Daily Advertiser from 19141918 and 1939-1945 were more discoloured in terms of yellowing than periods before or after. This was suggested to be due to the papers being accessed more times for these periods relating to retrieval of wartime narrative. The aim of the work was to use the functionality readily available and free to iPhone users to test the hypothesis posed by the archivist and then cross validate the findings with a calibrated colour measuring probe that is less readily accessible but provides a gold standardisation to test the efficacy of the smartphone. By using the simplest methods of testing and the conversion of the data from a free colorimeter app to a yellowing index devised for the experiment, the aim was to lower the barriers and expertise needed to make a stand alone meaningful determination, whilst also demonstrating if such activities could be adopted with future work, and protocols developed in order to emulate citizen based studies in other archives and collections.

Cellulose is our most abundant biopolymer produced by plants and bacteria. It has been utilised by humans for food, refuge, fuel, clothing and as a writing medium [7]. The method of artisanal paper production remains relatively unchanged whilst innovation in mass production has allowed paper to be the main storage medium for information until more recent challenges of the digital era. At the time of production little attention was paid to the longevity of paper; where the issues of paper deterioration in terms of fragility, hornification and yellowing now are significant [8]. The focus of the work here is on the yellowing of paper which the literature reveals to have a number of causes that are extrinsic and intrinsic [9-12], The yellowing of paper is seen as a challenge to conservation, where yellowing is synonymous with ageing and often more brittle paper which is especially problematic for archival access, and therefore also is an indicator of deterioration of paper integrity although there is a lack of correlation of discoloration with brittleness.

A literature search revealed a paucity in systematic longitudinal studies of paper yellowing within a single archive or groups of archives, elements of the Identical books project IBP [13] are probably the best known example. The opportunity to conduct a study for a single journal type that has been in continuous print for nearly a century and a half could allow the measurement and progression of yellowing in situ to be measured and the notion of further discoloration due to access of wartime newspapers tested. It was pointed out at the time of discussion-by the author-that change in yellowing of journals printed at wartime, if true, may be due to changes in paper production and sourcing at that time.

\section{Methods \\ Source material}

The Charles Sturt University Regional Archives and University Art Collection is a combined-function repository on the South Campus of Charles Sturt University in Wagga Wagga, NSW. The Archive has been the repository for material from the Riverina and Murray regions of NSW since 1973 and the Archive Collection now extends to over 4500 linear metres. The Riverina Archive storage of the Daily Advertiser 1876-2010 is in two areas of the archive 1876-1964 and 1965-2010 and has been consistently so for over 30 years. The arrangement of the archive and the state of some editions of the journal are shown in Fig. 1.

\section{Industry standard instrumentation}

The reference instrument used here is a spectroradiometer X-Rite I1 Pro, which measures the spectral range in $380-730 \mathrm{~nm}$ in $10 \mathrm{~nm}$ steps and uses an industry standard formula to calculate the red, blue and green response. The X-Rite I1 Pro has an optical aperture of $4.5 \mathrm{~mm}$, spectral resolution of $10 \mathrm{~nm}$, physical sampling interval of $3.5 \mathrm{~mm}$ and uses an internal simulated gas filled tungsten type A source, that can be compensated to mimic other light supplies. The X-Rite I1 Pro was used in reflectance mode with 3 data points measured 


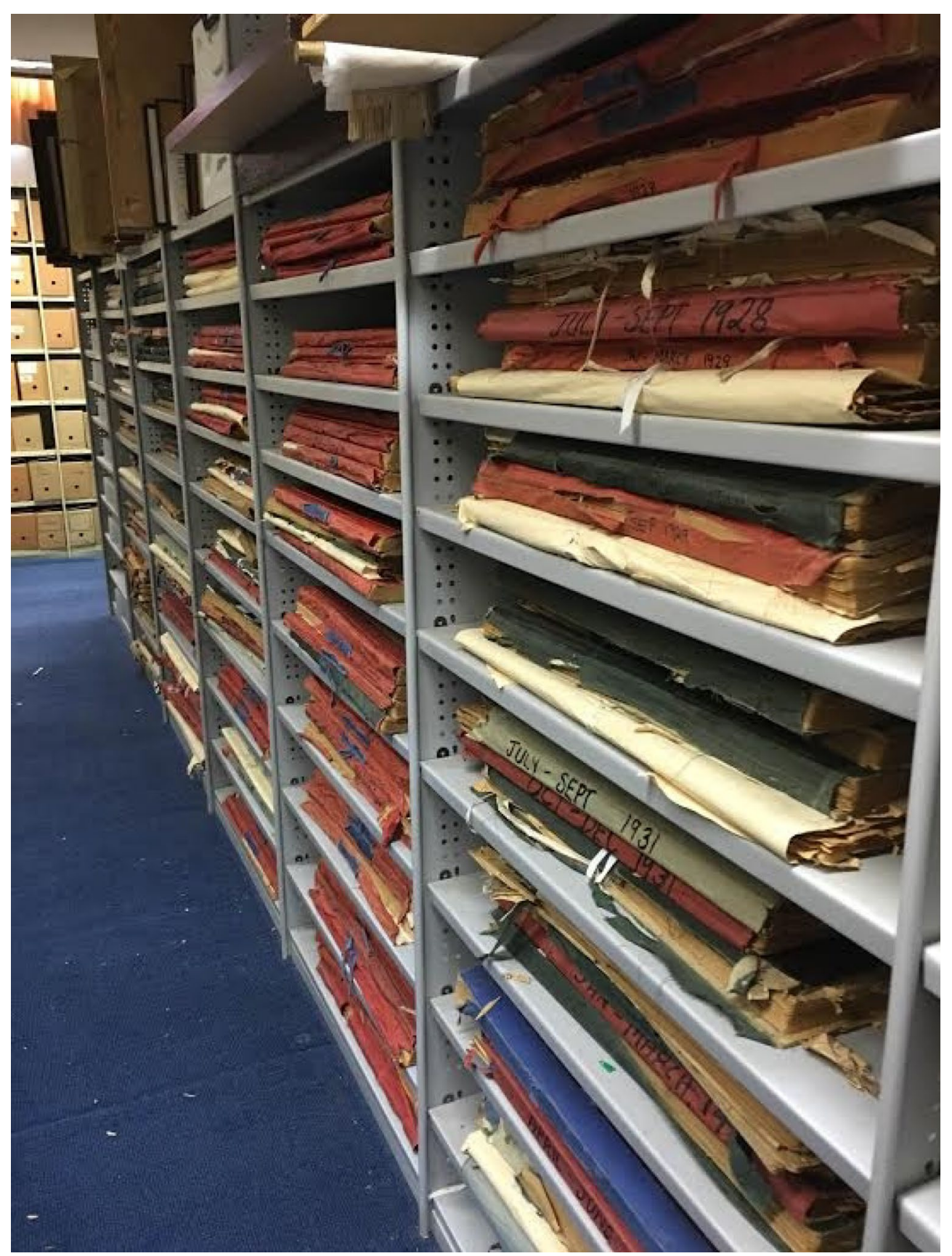

Fig. 1 The shelving arrangement of the Wagga Wagga Daily Advertiser 1876-1950. Journal articles from 1960 to 1972 were stored on the opposite facing shelves. More recent Journals to present day were stored on the ground floor in similar shelving. The rather brittle nature of editions especially from 1912 to 1950 mean that the sourcing and adjustment of the journals was easier for the measurements with the conventional spectroradiometer and only a smaller sample of journals were used for smartphone measurements

and averaged at each location used on the archive samples. The hand held device is placed in contact with the paper using a Perspex placement guide to make each measurement at a defined position. The calibration of the spectroradiometer for usage in each experiment used a whitened polypropylene blank as supplied with the equipment. The equipment itself is calibrated and certified by the manufacturer. 


\section{Stand-alone citizen science equipment}

The data collection for the citizen science scoping used an iPhone6S and an iPhone6 the specification of the integral camera is 8 megapixel $\mathrm{f} / 2.2$ with phase detection and autofocus to $29 \mathrm{~mm}$. The free colorimeter app the "Digital color picker 2 professional version 2" was downloaded from the iapp store. The app is developed by Jan Winter Eherenbergstrasse 310245 Berlin. In this, the colour is measured within the circle displayed in the field of view, and the measurement is captured by pressing the button in the display. The app offers the ability to use Automatic white balancing (AWB) that can be used to produce a point of calibration on a white source such as a piece of whitened polypropylene (the X-Rite I1 Pro calibration medium) then taking measurement with the AWB subsequently locked.

The output of the colorimeter is able to represent colours captured from images in a variety of formats. RGB/ RGB hex, CMYK, HSL/HSV, XYZ, CIE xy. These are output measures for different standards of colour, the CIE xy values form the axes of the human perception of colour according to CIE 1931 xy chromaticity [14] and are the parameters used here, although all are recorded simultaneously. The app developers claim to have measured the accuracy of the colorimeter by comparison to an industry spectroradiometer and found reliability to be within $5 \%$. The app does not produce the $\mathrm{L}^{*} \mathrm{a}^{*} \mathrm{~b}$ * more commonly used in yellowing measurements, and a proxy for yellowing had to be defined.

\section{Trial calibrations with smartphone}

The chromatic value $\mathrm{L}$ xy output from the iPhone6S was chosen as the parameter to compare to the $\mathrm{L}$ xy output of the X-Rite I1 Pro, furthermore, the value of SQRY $\left(x^{* * * 2} 2+y^{* * *} 2\right)=Y$ can be used to evaluate a degree of yellowing $(\mathrm{Y})$ from both instruments corresponding to a path of increasing yellowness in the CIE chromaticity two dimensional plot [14]. The measurement of identical proxy journal samples indicated that the light source, the AWB balance on the smartphone and sample to lens distance had the largest effect on L xy output. Trials were established for three archive samples exhibiting different degrees of yellowing. Both iPhones were held at distances between 7 and $1 \mathrm{~cm}$ at $1 \mathrm{~cm}$ intervals in a number of different lighting conditions as follows (1) natural sunlight indoors (2) fluorescent strip lighting of the archive (3) LED lamp at a distance of $10 \mathrm{~cm}$ (4) A second iPhone LED light held in a clamp allowed LED light to flood the axis of sample to lens. Results of the trial (not shown) indicated that the closest obtainable values to those of the $\mathrm{X}$-Rite I1 Pro, were obtained using illumination from a second $i$ Phone at right angles to the detecting iPhone (at a distance $6 \mathrm{~cm}$ from sample) with a detecting camera lens to sample distance of $4 \mathrm{~cm}$, irrespective of which iPhone was used as detector. This set up with AWB locked on the smartphone app was used in the subsequent sampling of the journal archive.

\section{Journal sampling}

A number of trail runs were assessed to examine the number of data points needed for both the iPhone6S and the X-Rite I1 Pro. The special protection afforded to the earliest journals meant that it was decided to commence the overall data collection from 1868 and to end at 2004 with sampling on average at two year intervals. Due to convenience of access whilst minimising damage, it was decided that a sample journal collection from each shelf (Fig. 1) would be used for the X-Rite I1 Pro, Access to a journal from one folder on each shelf meant that over 100 journals would be sampled by the X-Rite I1 Pro, the January-March folder being used for any year where possible. The sampling points typically used are shown in Fig. 2 with six points measured per page (effectively $2 \times 3$ triplicates). The outside vertical margin of the journal proved to be an effective sampling point, although this was sometimes more discoloured than the body of the journal it provided a consistent series of measuring points with no ink from the reverse side. Use of the margin for measurement would also accentuate any variation in level of journal use. In each folder two pages were sampled, one from the front of the folder and what was estimated to be the middle of the folder. This is a similar approach to that taken in the IBP [13]. It was quickly noticed that the first page of some journal folders was discoloured possibly due to the interaction with the cardboard of the folder and therefore "front page" sampling was made within the first five pages at the front of the folder. Figure 3 also shows the state of deterioration and yellowing of some journals where extra care had to be taken in measurements.

In order to address the hypothesis posed that the war editions were more discoloured due to extent of access, a more detailed investigation year by year was made with both colorimeter devices over the journal period of 1900-1920 using the protocols described above. This was also to test if a stand-alone testing of the hypothesis using a smartphone was possible. The setup for the illumination and measurement with iPhones is shown in Fig. 4.

All data sets collected on the X-Rite I1 Pro were exported from the devices to Excel where the combined xy vector length, as described above, was calculated to produce the value $\mathrm{Y}$ measurement approximating to yellowing in the perception of the human eye [14]. Data from the smartphone was converted to $\mathrm{Y}$ on the smartphone calculator in the spirit of developing a standalone device. We note that the standard presentation of 


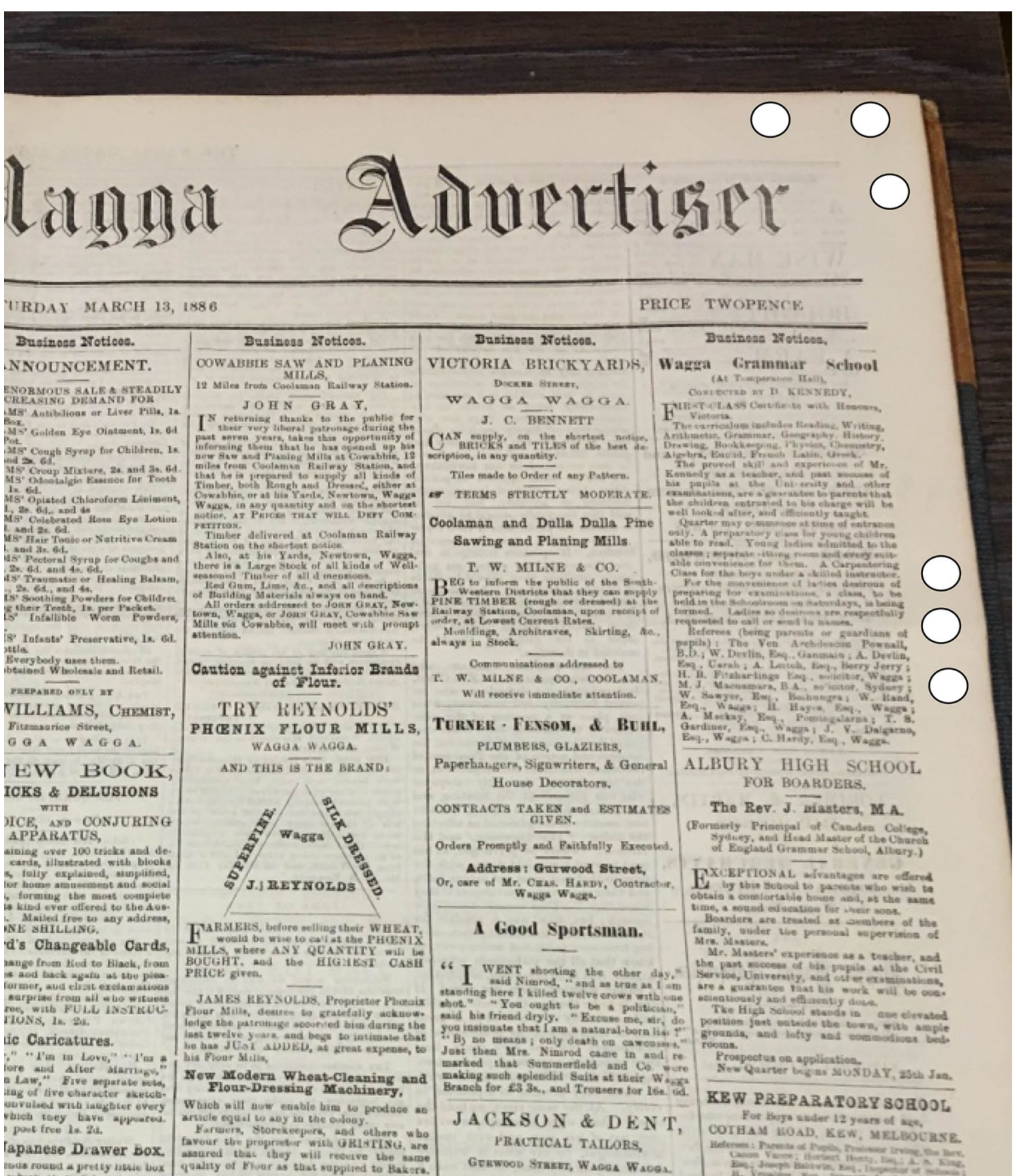

Fig. 2 Copy of the Wagga Wagga Daily Advertiser from 1886. The figure shows six white circles indicating the typical positions used to make two sets of triplicate measurements on a page close to the beginning and centre of each folder. Such sites were used for the X-Rite I1 Pro, with triplicate measurements made for the mid page section in fewer selected journals using the iPhone6S

yellowing in many articles relates to the $b^{*}$ value of the CIE $L^{*} a^{*} b^{*}$ however the $b^{*}$ value could not be directly obtained from the iPhone app and would require non linear manipulation as described in $[5,6]$. This would require an extra layer of processing complexity and loss of principle of the iPhone as a stand-alone device.

\section{Results}

The scatter plot of the xy data obtained from measurements on the journal using the X-Rite I1Pro as evaluated by being overlaid on the 2D gamut of the xy CIE 1931 values are shown in Fig. 5, this shows the distribution of the $\mathrm{x}$ and $\mathrm{y}$ values that correspond to the different visually 


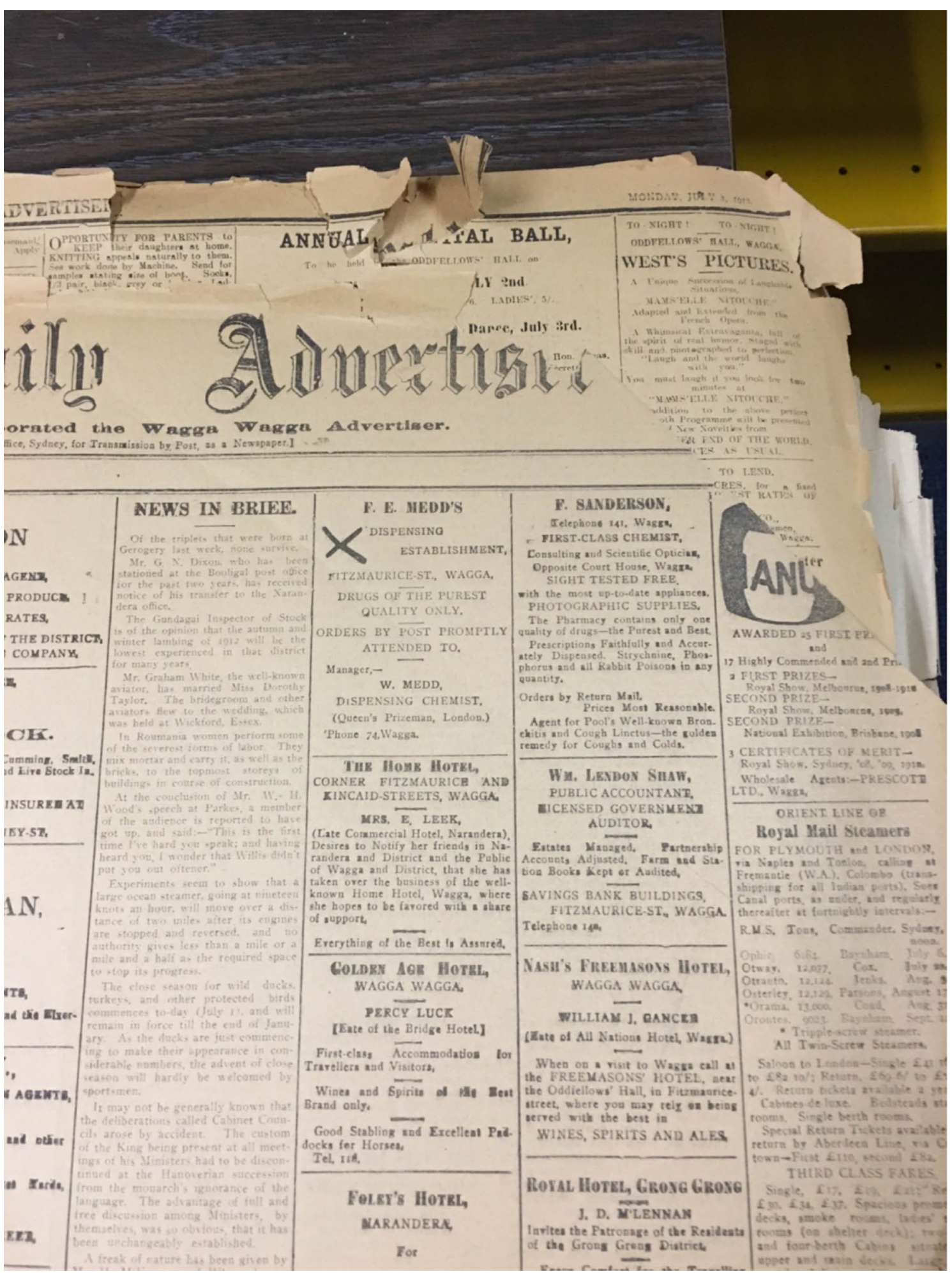

Fig. 3 Example of Wagga Wagga Daily Advertiser exhibiting yellowing and brittleness that prevented the first few leaves being easily sampled. This was a typical example of a journal showing damage that puts sampling and extraction from the archive at risk. It was always easier to locate a mid page margin section than a top corner. Although such brittle examples relate to yellowing, the degree of brittleness varied amongst journals where the yellowing effect appears to be saturated 


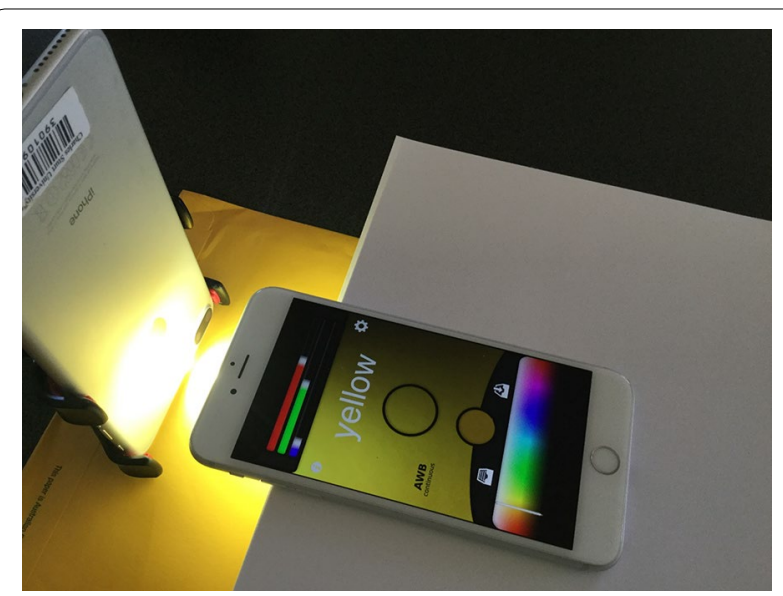

Fig. 4 The use of two iPhones as illumination source and detector on a piece of yellow paper. The illuminating iPhone is held in the vertical plane be a clamp and the detector iPhone is held as a sample distance by a stack of white cartridge paper

perceived hues. The clustering of the scatter plot along a line of increasing yellow hue indicates that the vector length product of the xy data corresponds well to the region of the visual perception chart that corresponds to

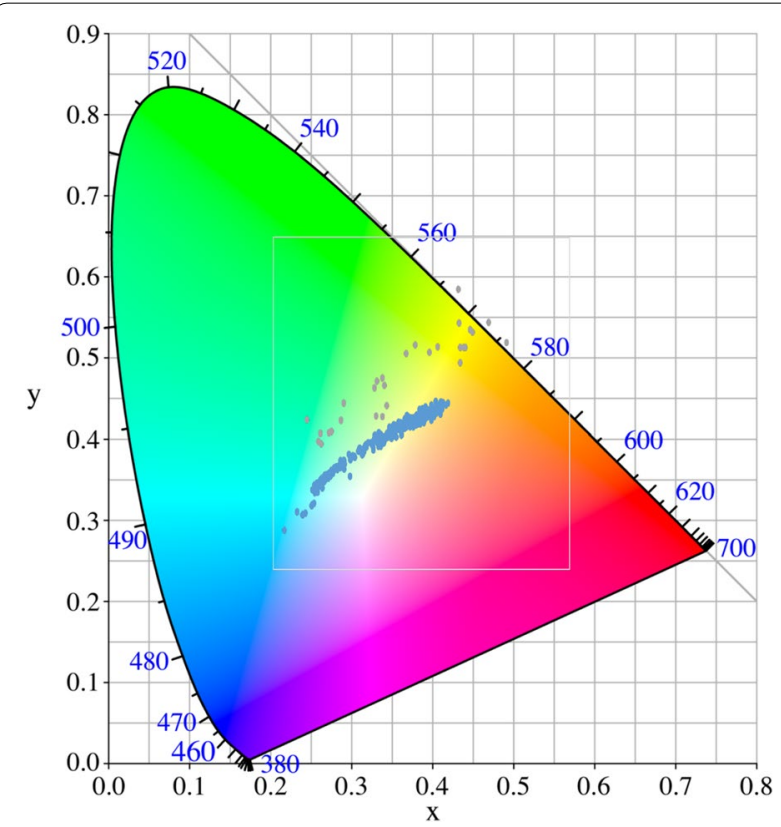

Fig. 5 A scatter plot of the xy values obtained from the journal collection. An indication that the vector length of the xy coordinate is sufficient to describe the yellowing. Blue numbers around the edge of the gamut indicate wavelengths of light. The data points obtained from the $X$-Rite 11 Pro are shown as blue squares and cover a range of the yellow gamut in the CIE xy 1931 colour range they are laid over. $X y$ values from the iPhone 6 s are shown as green circles. The vector product of $x y$ is used to determine level of yellowing perception of yellowing. The distance from the origin as a degree of yellowing is therefore suitable in this context to support the stand alone approach with xy measurements converted into the vector length yellowing index Y. The xy measurements for the iPhone6S sampling are also shown on the same figure and indicate that there is an offset of the measurements from the iPhone6S toward the green/blue compared to the industry standard. This may be due to the LED source being deficient in red light.

The results of the in situ longitudinal 1866-2004 study are shown in Fig. 6 for the large timescale. The plotting of four data points (two pages per folder at two margin locations in triplicate) for each year sampled, shows the variability of measurement as a further indicator of change in the document. The Figure contains three distinct areas of trend with respect to print date of the journals, (1) the period from 1868 to 1912 where the journals are some of the least yellow observed until those of the contemporary period of 1996. (2) The period of 1912-around 1968 corresponds to a more widely distributed series of $Y$ values but correspond overall relatively flat region suggesting saturation and finally (3) a decrease of yellowing vector from $\sim 1960$ to 2004, where the date of the journal seems to be the main determinant.

Figure 7 shows measurements of the yellowing Y using both the iPhone and the X-Rite I1 Pro to investigate the yellowing of journals around the period of WWI. These indicate a change in the yellowing as measured from 1900 to 1920 with sampling at 1 year intervals covering the WWI period. The measurement by the smartphone is more variable than that of the X-Rite I1 Pro, but does show the trend in Y especially the step change at 1912, however both techniques do not show an increase in $\mathrm{Y}$

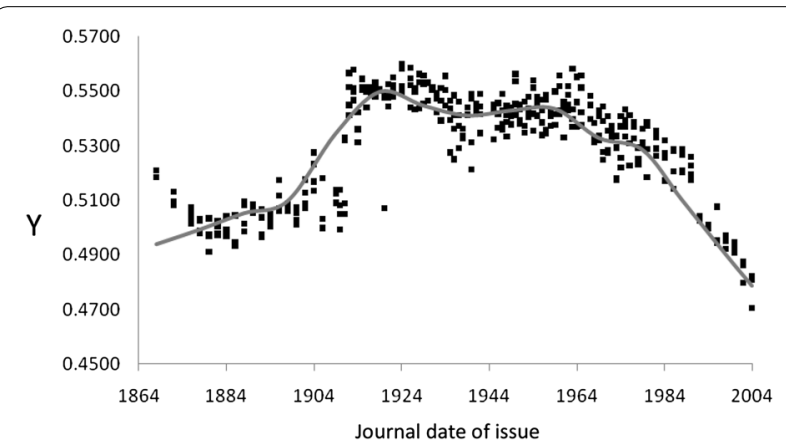

Fig. 6 Graph of yellowing vector $Y$ measured against print date of journal. Black squares the vector length of the product $x y$ from measurements, the Yellowing parameter $Y$ (Y-axis) to give an indication of relative time dependent yellowing of the journal date of issue ( $X$-axis) of the Wagga Wagga Daily Advertiser from 1868 to 2004 using the $X$-Rite 11 Pro. The main regions of distinction can be seen; these are described in the "Results" section. The 10 year rolling average is shown as a grey line 


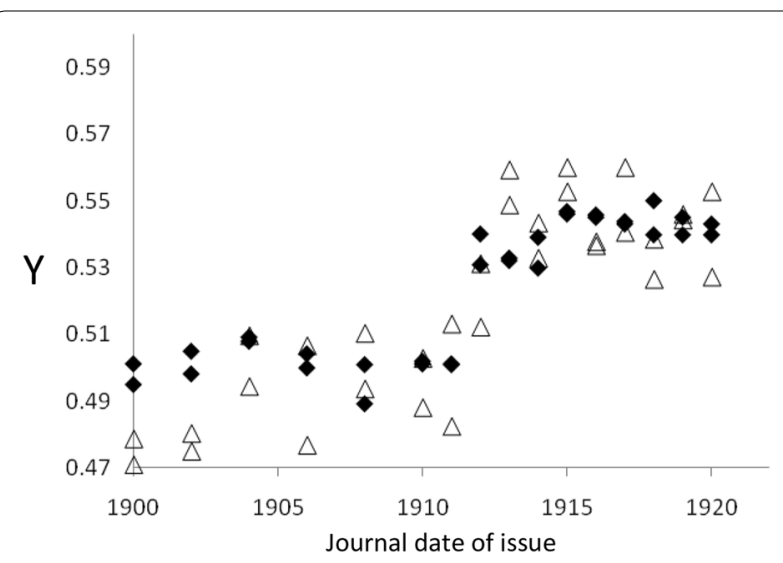

Fig. 7 Measurement of Y 1900-1922 with the iphone6S and X-rite /1 Pro. iPhone6S (White triangles) and X-Rite 11 Pro (Black diamonds) values of the yellowing parameter $Y$ plotted as a function of journal date of issue 1900-1922. The values from the smart phone were found to systematically report a higher value and a global correction values of 0.94 was found to optimally give an overlay of the values on to those of the gold standard. Both detection methods show the stepwise increase in $Y$ in 1912 however the assertion that the $Y$ value was higher for wartime journals specifically is not proven

specifically corresponding to the war years 1914-1918. The results used with the smartphone illumination showed a Y value that was systematically higher than that of the gold standard device, a correction factor of 0.94 applied to all the smartphone $\mathrm{Y}$ values gave an optimal overlap of both datasets.

\section{Discussion}

\section{Citizen science and crowdsourcing at large}

The definition of crowdsourcing is paraphrased here as an outsourcing of a function by a company or institution to an undefined and generally large network of people in the form of an open call [15]. The information flow from the almost ubiquitous internet has developed a "cognitive surplus" [16], that manifests itself in behaviours that are; passive (watching, listening) or active (blogging, tagging, reviewing). Citizen science can be described as a subset of such activity where the scientific method is essential to allow crowdsourced information or measurements to be validated as evidence within the commonly held framework of scientific understanding. The Citizen Science Alliance requires that its projects answer a real scientific question [17]. Citizen science growth will be inextricably linked to technologies in the ability to obtain data from multiple spatial and temporal scales, gaining efficiencies in data collection, processing and data management, whilst adapting and adopting technologies to allow participants and organizers to communicate and interact effectively $[1,18]$. Citizen science of note is often shepherded or co-ordinated by an experienced scientist who is reaching out to the public in order to increase a voluntary workforce and also to improve the understanding of science and its impact in communities. Citizen science programmes such as Galaxy Zoo [19, 20], have produced data analysis by members of the public invited to categorise images of galaxies from telescope images. These analyses have been used in publications, however the process does contain a number of fail-safe procedures so that untrained behaviour does not intervene in the good will of the many.

\section{Citizen science and crowd sourcing in cultural heritage}

Galleries, Libraries, Archives and Museums (GLAMs) are involved in crowdsourcing and citizen science projects, however the number is fewer than that found, for example, in the biodiversity arena. The backlog of unresearched material in GLAMs can provide a valuable framework for data gathering, cataloging and digitisation and also can be used as a form of public engagement with cultural heritage, the concept of the citizen historian is already well established [21]. The nature of GLAMs also speaks to the ability of the resources to investigate humanities based research questions as well as citizen science activities [22]. The majority of citizen based interventions are contributory [1] related to tagging, transcribing, or interrelating cultural items. Within cultural heritage citizen science, the number of projects described becomes far fewer when measurement of data as opposed to collection or sorting of data is described, this is possibly because of the extra training or co-design needed to be more sophisticated above recognition, identification or transcription through "human computation" [16] which captures many of the extant activities.

\section{Mobile technology}

The emergence of precision hand held commonplace technology may mean that more involved scientific measurements could be made by citizen scientists with the affordable everyday technology such as camera drones, digital cameras and smartphones to capture images, sound and digital data. Such activity can be described as complementary [23] where information collected can be added to a resource such as the UK soundmap where sounds can be recorded on a smartphone using an app called Audioboo tagged with metadata and placed by the British Library on a map [24].

\section{Smartphones as a colorimenter in research}

The use of smartphones as suitable instruments for data collection is growing [23, 24]. The development of the smartphone as a stand-alone optical sensing tool for different applications with water samples such as fluoride 
[25] metal pollutants [26] or pesticides [27], requires a step of chemical reaction to produce a detectable colour. A review of smartphone biosensors [28] shows their utility at point of care and point of need to make bioassay measurements especially in rural and remote regions. The research described above has also shown that the variability of measurement within a smartphone type is small.

Within cultural heritage, citizen science that utilises a smartphone is a small subset of the digital image analysis and identification tasks that are employed at GLAMs. The use of the citizen scientist's own technical resource is a further point of distinction where selection, data collection and digitisation is made by the citizen scientist. Within this subset of digital processing of cultural heritage is the use of colorimetry to measure change in collections. Two papers $[5,6]$ describe the proposed scoping activity of crowd sourced images to monitor built heritage through colorimetry. The popularity of digital photography on smartphones across the world gives access to a mass of images that can be processed to colorimetric data by the use of a calibrant chart at each site of interest. These deal with the built environment although the proof of principle for the work reported was on observation of the colour change in a cut potato! Here the images from a mobile phone are transferred to a PC for extensive processing to combine the (nonlinear) sRGB to CIE L"a*b* using Matlab processing. The images can then be described as a single value such as $b^{*}$ to show that smartphones have a place in the measurement of on-going change in the built environment. The approach, although powerful, therefore does not give the standalone approach sought in this project.

\section{The suitability of a smartphone as a stand-alone device to test a hypothesis from cultural heritage?}

The challenge of this project was to test if data obtained on a smartphone (with one off validation) without any further need for additional supporting technology, could test a cultural heritage scientific hypothesis. The use of a free app with subsequent relatively trivial calculation of vector length $\mathrm{Y}$ on the iPhone calculator to convert the colorimeter data into a measurement of yellowing was adopted as the main challenge of the work presented here. The calibration with an industry standard colorimeter was used to optimise the way in which the iPhone was used and since comparable results were obtained to the X-Rite I1 Pro as shown in Fig. 7 with the step change in colour at 1912, therefore it was demonstrated that the iPhone could now be used (with caution) independently to assess yellowing of historical newsprint and test the hypothesis. The autonomous use of the iPhone without the need to post process the data on a PC, or to have the
iPhone simply used as a data capture device with extensive expert post processing, could expand the ability of the public to become part of more extensive studies, this of course would also require specific design principles. Therefore although the study was relatively limited, it had the purpose to answer a valid query from an archivist and the more extensive data collection also revealed an interesting longitudinal study of newsprint yellowing measurements in archival material for a period over a longer time span than the IBP (whilst borrowing sampling methodology from it).

It was also noted that from the measurements used in the IBP p66 "Developing a cadre of conservators to undertake and carry forward this research has been rewarding. Ensuring that they formed a coherent team that communicated without restraint internally and with the external experts was essential. Bringing the group together for joint training sessions helped in this, as did enabling them to travel to each others' libraries and to research laboratories" [13]. The training of the user is therefore paramount in citizen science.

From the foregoing, the quality of the camera and the sophistication of the app is of a capability that may eventually outstrip the standard bench or portable devices available until recently, however the basis for the systematic differences observed need to be determined so that measurements can be made in a variety of archival contexts with validity. It is noted that the digital negative format (.dng) that is available on some Android phones may allow the raw data images to be obtained without internal process.

Caution is therefore needed, the capability of the device needs to be married with the prudent and planned employment by the user, or a team of users. The next steps based on the determinations made here can be focussed on the use of the approach to investigate other artefacts in terms of change of colour and also to consider the level of expertise needed to obtain reliable results that are comparable between users. This article will be used as a point of focus to garner interest in the Riverina region to talk to local historical societies about the information beyond the written word in the archives and the vigilance needed in measurement of change where citizen scientists can contribute.

The stand-alone approach taken here is an early indicator of the capability of detection and computation that is close at hand. Similar analyses such as Fast Fourier transforms which are available as apps, may expand the capability of stand-alone analysis especially in remote settings where analysis is needed even when the internet and PC are not available. In a country where the tyranny of distance is a major challenge, these options for immovable cultural heritage may prove to be useful. 
Measuring change of documents is a major challenge in archiving and conservation especially in understanding the spectral changes involved with paper degradation [29]. Paper yellowing is a major identifier of ageing in archive collections [30, 31]. In part it provides character to collections and is an important factor of the public perception of historical storage such as is the smell of libraries and archives [32, 33]. The yellowing can become problematic especially in fine art drawing rather than newsprint where text can often still be read. Relatively few studies have been made of longitudinal nature such as this presented here, however the IBP [13] p31 has a similar intent to measure the changes in identical books where measurements were made ".... at two locations on one page, using a Konica CM-2600d reflection spectrophotometer controlled by SpectraMagic software on a laptop computer Colour measurements were taken close to the external top right corner and at a position in the middle of the page with no printing on either side of the leaf. Each measurement was the average of three small area replicate measurements". The range of measurements obtained from the British Library Identical Books shows considerable scatter of yellowness as a function of age. The general trend in the IBP reveals that from 1900 to 1950 a relatively static level of yellowing with $b^{*}$ around 13 which begins to decrease from around mid 1950. This exhibits a strong similarity to the data reported here in Fig. 6 albeit with different yellowing indicators. The IBP however did not contain data from the period of 1860 s-1900 which indicated in the study here that a different level of yellowing was detected.

In examining the variation of the yellowing parameter $\mathrm{Y}$ with respect to time, we can draw a number of conclusions. Firstly, there is a non linear and complex relationship between the yellowing of paper and time in this case. The generally accepted statement that paper yellows with time is not held to be true and that possibly, the composition of the paper was far more a determination of yellowing. A simple visual inspection showed the change of paper from pre and post 1912, however the subtle changes in pre-1912 samples observed in the two regions (1868-1880 and 1880-1912) would probably not be picked up by eye. This region suggests change in medium or storage pre and post 1880 . The initial paper used for the Daily Advertiser appears to have maintained a low yellowing level and also is relatively robust as copy. As soon as journals post 1912 print date were studied, previous access indicate damage to many copies, with fragments of newsprint present in the folders as change has occurred. Although this is not a multifactorial study of paper properties, it was observed that the brittleness of paper also changed discontinuously as a function of time. A visual examination showed the journal newsprint from 1912 to 1930 exhibiting the greatest fragility. Both these observations point to a change in the use of the paper source and variation in paper source and processing over time.

The most discoloured journals equate to dates of 1924 and 1963, neither relate to any major historical events of Australia or the Riverina region. Enquiries at the Daily Advertiser office were unable to shed light on the use of paper source or changes although this article may help to draw community information on this matter. The plateau region of yellowing vector Y from 1912 to 1964, may point to a saturation in the yellowing condition possible with this type of paper, we are unaware of saturation being reported in an archive context previously. This factor of colour saturation may be a shortcoming of using yellowing to indicate degradation since further mechanical degradation of newsprint could occur after the point of discoloration saturation is reached [9]. The variability of the values measured at the 4 points in the journal volume indicate that the sampling from the front pages and middle pages may mean that the overall saturation is not complete in each folder. From 1960 to 2004 the yellowing vector is an inverse function of print date. It is of interest that the paper of the journals 1880-1910 are comparable in yellowing to that of journals from 1996. This is presumably because a non acid extracted paper was used until 1912 and the lignin richer pulp used after this point was more susceptible to yellowing.

It is of interest to note that the original supposition that the journals pertaining to the times of two World Wars may be more yellow due to more frequent access does not hold.

\section{Conclusions}

This manuscript evaluates the use of an industry standard portable spectral device and smartphone to determine a longitudinal study of the changes in paper discoloration in a single journal type in a single archive. The work clearly shows that the type of paper used is a major determinant in yellowing that is greater than age alone. The comparison with a more accessible device indicates that such accessible technology could be used in citizen science but would need to be carefully controlled. In addition, consideration needs to be made of the impact that well intended data collection could result in, for both improved understanding of an archive content and potential damage without meaningful data production.

\footnotetext{
Acknowledgements

I would like to thank the following people for their help and support in this project. Peter Ryall of the Faculty of Science office for data handling. Gail Fuller and Gary McKenzie of SPAN for the loan of equipment and guidance on measurement. Wayne Doubleday and Julia Kohlhagen for help in access to the Charles Sturt University Riverina Archive.
} 


\section{Competing interests}

The author declares no competing interests.

\section{Publisher's Note}

Springer Nature remains neutral with regard to jurisdictional claims in published maps and institutional affiliations.

Received: 9 November 2016 Accepted: 13 July 2017

Published online: 21 August 2017

\section{References}

1. Bonney R, Cooper CB, Dickinson J, Kelling S, Phillips T, Rosenberg KV, Shirk J. Citizen science: a developing tool for expanding science knowledge and scientific literacy. Bioscience. 2009;59:977-84.

2. http://www.ala.org.au/get-involved/citizen-science/.

3. Kohle H. Social tagging of artwork or: how do I teach young people to go to the museum. Mus News. 2009;161:14-5.

4. Kohle H. Art history goes social media. Lay optimize an image database-a digital game. Aviso J Sci Arts Bavaria. 2011;3:37-43.

5. Ma W, Walton M, Cossairt O, Bearman G, Doehne E. Crowd-sourced mobile phone images for heritage conservation monitoring. In: Digital Heritage, vol. 1. New York: IEEE; 2015. p. 111-4.

6. Bearman G, Doehne E, Voss J, Merrill K, Bagaria R. Citizen science and mobile phone cameras as tools for monito-ring world heritage. Built heritage monitoring conservation management. 2013. p. 737-44.

7. Hunter D. Papermaking: the history and technique of an ancient craft. New York: Dover Publications; 1978.

8. Kato KL, Cameron RE. A review of the relationship between thermallyaccelerated ageing of paper and hornification. Cellulose. 1999:6:23-40.

9. Carter HA. The chemistry of paper preservation: part 2. The yellowing of paper and conservation bleaching. J Chem Educ. 1996;73:1068-73.

10. Daniels V. The discolouration of paper on ageing. Paper Conserv. 1988;12:93-100

11. Hon DN. Yellowing of modern papers. Preservation of paper and textiles of historic and artistic value II. Washington: American Chemical Society; 1981. p. 119-41.

12. Conte AM, Pulci O, Knapik A, Bagniuk J, Del Sole R, Lojewska J, Missori M. Role of cellulose oxidation in the yellowing of ancient paper. Phys Rev Lett. 2012;108:158301.

13. Knight $B$, Horie $V$. The identical books project. Int Preserv News. 2007:42:18-22.

14. Smith T, Guild J. The CIE colorimetric standards and their use. Trans Opt Soc. 1931;33:73-134.

15. Dawson Ross Crowdsourcing landscape http://crowdsourcingresults. com/competitionplatforms/crowsourcing-landscape-discussion.

16. Shirky C. Cognitive surplus: creativity and generosity in a connected age. New York: NY penguin press; 2010.
17. Romeo F, Blaser L. Bringing citizen scientists and historians together. In: Trant J, Bearman D, editors. Proceedings of museums and the web. 2011. http://conference.archimuse.com/mw2011/papers/bringing_citizen_scientists_historians_together. Retrieved 16 July 2014.

18. Cohn JP. Citizen science: can volunteers do real research? Bioscience. 2008;58:192-7.

19. Lintott CJ, Schawinski K, Slosar A, Land K, Bamford S, Thomas D, Raddick MJ, Nichol RC, Szalay A, Andreescu D, Murray P. Galaxy Zoo: morphologies derived from visual inspection of galaxies from the Sloan Digital Sky Survey. Mon Not R Astron Soc. 2008;389:1179-89.

20. Wiggins A, Crowston K. From conservation to crowdsourcing: a typology of citizen science. In: IEEE, system sciences (HICSS) 44th Hawaii international conference. 2011. p. 1-10.

21. Ridge M. From tagging to theorizing: deepening engagement with cultural heritage through crowdsourcing. Curator Mus J. 2013;56:435-50.

22. Dunn S, Hedges M. "Crowd-sourcing scoping study. Engaging the crowd with humanities research." Centre for e-Research, King's College London. 2012. http://crowds.cerch.kcl.ac.uk/wp-uploads/2012/12/Crowdsourcingconnected-communities.pdf.

23. Oomen J, Aroyo L. Crowdsourcing in the cultural heritage domain: opportunities and challenges. In: ACM proceedings of the 5th international conference on communities and technologies. 2011. p. 138-49.

24. http://sounds.bl.uk/uksoundmap/index.aspx.

25. Levin S, Krishnan S, Rajkumar S, Halery N, Balkunde P. Monitoring of fluoride in water samples using a smartphone. Sci Total Environ. 2016;551:101-7.

26. Wei Q, Nagi R, Sadeghi K, Feng S, Yan E, Jung Ki S, Caire R, Tseng D, Ozcan A. Detection and spatial mapping of mercury contamination in water samples using a smartphone. ACS Nano. 2014:8:1121-9.

27. Mei Q, Jing H, Li Y, Yisibashaer W, Chen J, Li BN, Zhang Y. Smartphone based visual and quantitative assays on upconversional paper sensor. Biosens Bioelectron. 2016;75:427-32.

28. Roda A, Michelini E, Zangheri M, Di Fusco M, Calabria D, Simoni P. Smartphone-based biosensors: a critical review and perspectives. TrAC Trends Anal Chem. 2016;79:317-25.

29. Missoria M, Pulcia O, Conteb AM. Spectroscopy of Ancient Documents. London: Springer; 2015.

30. Sobucki W, Drewniewska-Idziak B. Survey of the preservation status of the 19th and 20th Century Collections at the National Library in Warsaw. Restaurator. 2003:24:189-201.

31. Padoan R, Steemers TA, Klein ME, Aalderink BJ, De Bruin, G. Quantitative hyperspectral imaging of historical documents: technique and applications. In: Proceedings of the 9th international conference on NDT of art, Jerusalem Israel, 25-30 May 2008. http://www.ndt.net/article/art2008/ papers/097Padoan.pdf.

32. Bembibre C, Strlič M. Smell of heritage: a framework for the identification, analysis and archival of historic odours. Herit Sci. 2017;5:2. doi:10.1186/ s40494-016-0114-1.

33. Ferro S. FYl: why do libraries have that smell. Pop Sci. 2012;19.

\section{Submit your manuscript to a SpringerOpen ${ }^{\circ}$ journal and benefit from:}

- Convenient online submission

Rigorous peer review

- Open access: articles freely available online

- High visibility within the field

Retaining the copyright to your article

Submit your next manuscript at springeropen.com 